\title{
Optimizing the performance of magnet-controlled capsule endoscopy based on radiological and gastroscopic modeling
}

\author{
TING-JI SUN, CHUN-SHENG CHENG and HOU-DE ZHANG
}

Department of Gastroenterology, Nanshan Hospital, Guangdong Medical University, Shenzhen, Guangdong 518052, P.R. China

Received September 4, 2018; Accepted October 9, 2019

DOI: $10.3892 /$ etm.2019.8202

\begin{abstract}
Routine use of magnet-controlled capsule endoscopy of the stomach has been limited by the inadequate views of specific stomach regions. In the present study, radiology and upper gastrointestinal endoscopy (UGIE) were used to determine optimal subject body positioning and suitable external control magnet placement for capsule endoscopy. Healthy adult volunteers were subjected to upper gastrointestinal X-ray radiography $(n=5)$, spiral computed tomography with volume reconstruction $(n=4)$ or UGIE $(n=1)$. Stomach fundus-to-body (FB) and body-to-antrum (BA) angles were compared when subjects were supine, prone, lying on their left side and on their right side, and when they were standing upright. Vertical distances from the surface of the body to the distal points of the fundus and antrum were also compared in this range of subject positions. Obtuse angles were considered the most beneficial for capsule movement and short vertical distances were considered desirable for optimizing magnetic force. The FB angle was sharply acute in the supine position, relatively open where subjects were on their side, and almost $180^{\circ}$ in the standing position. The BA angle was obtuse in the standing position but acute in all other positions. With the subject in any position, the left lower lateral chest had the shortest distance to the fundus, while the ventral wall was closest to the antrum. The present modeling analysis indicates that standing is superior to all decubitus positions for magnetic-capsule endoscopy, including the commonly used supine position. Both the abdominal anterior wall and left lateral lower chest appeared to be advantageous locations for external control magnet placement.
\end{abstract}

Correspondence to: Dr Hou-De Zhang, Department of Gastroenterology, Nanshan Hospital, Guangdong Medical University, 89 Taoyuan Road, Nanshan, Shenzhen, Guangdong 518052, P.R. China

E-mail:szkjk@126.com

Key words: magnet-controlled capsule endoscopy, body positioning, external control magnet placement, standing position, gastric angles

\section{Introduction}

The invention of a wireless capsule endoscope by Israeli scientists in 2000 not only ended the long history of visual inaccessibility of the small intestine, but also brought about a new era of painless minimally invasive gastrointestinal endoscopy. Shortly thereafter, capsule esophagoscopy and capsule colonoscopy were developed (1-4). First-generation capsules are propelled by gastrointestinal peristaltic movement, which makes them ill-suited for use in thorough examination of the wider area of the stomach.

In 2010, the same Israeli scientists reported the first use of a magnet-controlled capsule endoscope in the stomach of a young male volunteer. Under synchronous observation of traditional upper gastrointestinal endoscopy (UGIE), the capsule was demonstrated to move precisely and rapidly to a designated location in response to the movement of a handheld external magnet around the torso of the volunteer (5). Subsequent studies attempting to replicate the success of that first trial have been unable to recreate the flexibility of manipulation described, regardless of whether the magnetic capsule was manipulated with a handheld magnet or a robot-assisted magnetic manipulation system. Furthermore, operators have had difficulty achieving complete exploration of the stomach (i.e., without any blind areas), especially of the gastric fundus and cardia (6-11). In China, a magnet-controlled capsule specialized for the stomach has been approved for clinical use, but has also failed to solve the aforementioned problems $(12,13)$.

Analysis of the differences between the first trial and subsequent reports revealed methodological differences, which may be responsible for the inconsistent findings. The first trial was conducted with the volunteer lying on his left side, whereas the majority of the subsequent studies were conducted with subjects lying in a supine position. Additionally, in the first trial, gastric distention was maintained by continuous gas injection in association with concomitant UGIE, whereas in subsequent studies, gastric distention was achieved by pre-examination drinking of water and gas producing agents. Thus, the different findings between the first and subsequent studies may be due to difference in the subjects' body positioning, the extent of gastric distention, and potentially the distance between the internal capsule and the external magnet.

Rahman et al (8) used computed tomography (CT) modeling of the abdomen to determine the optimal placement 
of a magnetic capsule endoscope in the stomach, with respect to enabling complete mucosal visualization, and to determine the optimal placement of the handheld magnet for traversing the pylorus. Maximal visualization (85\%) was achieved with a combination of two stations at opposite ends of the stomach, one at the fundus and one at the antrum. The optimal magnet position for traversing the pylorus posteriorly was found to be between vertebrae T5 and L2. However, the inability to achieve $100 \%$ visualization of the fundus and cardia with any station combination remains a major challenge.

The aim of the present study was to examine the influence of body positioning and magnet-capsule distance on intragastric navigation of the capsule, an area of research that has received limited attention $(7,8)$. Radiology and UGIE images were examined to compare gastric morphology between subjects in different body positions with the aim of determining optimal subject body position and external control magnet placement in order to enable magnet-controlled capsule endoscopy with a thorough exploration of the stomach.

\section{Materials and methods}

Subjects. In total, 10 healthy adult volunteers [age, 30-49 years; body mass index (BMI), 20.7-24.6] were enrolled between 1st March and 1st April 2018 at Nanshan Hospital, Guangdong Medical University (Guangdong. China) including 5 subjects ( 2 men and 3 women) who received double-contrast barium upper gastrointestinal X-ray radiography (UGI-XR), 4 subjects ( 2 men and 2 women) who received virtual anatomical stomach modeling by spiral CT with a volume rendering technique (VRT), and 1 male subject who received traditional UGIE. The inclusion criteria were as follows: i) Age range, 20-60 years; ii) no pregnancy planed within six months; and iii) signed written informed consent. The exclusion criteria were as follows: i) Overweight $(\mathrm{BMI}>25)$ or underweight $(\mathrm{BMI}<19)$; ii) liver and kidney dysfunction; iii) afflicted with other gastrointestinal diseases, including gastrointestinal bleeding or intestinal obstruction; and iv) exhibited allergy to contrast agent.

The study protocol was approved by the Independent Ethics Committee of Nanshan Hospital, Guangdong Medical University. Written informed consent was obtained from every volunteer.

UGI-XR. UGI-XR was conducted according to routine clinical protocol. The subjects were instructed to fast overnight to ensure at least $8 \mathrm{~h}$ of fasting before the examination. At $10 \mathrm{~min}$ post-oral administration of a spasmolytic agent (10 mg anisodamine, Minsheng Pharmaceutical Co., Ltd.), 3 g gas-producing agent powder (citric acid, sodium bicarbonate 1:1, Bosen Pharmacy Co., Ltd.) was administered orally, followed by rapid drinking of $200 \mathrm{ml}$ barium (220\% weight/volume). For each subject, eight X-ray images of the stomach were captured with a DR Definium 6000 (GE Healthcare) as follows: Supine decubitus (1 image), prone decubitus (1 image), right lateral decubitus (1 image), left lateral decubitus (1 image) and standing upright (4 images). The standing upright images included a ventral-facing image, a dorsal-facing image, a left-facing image and a right-facing image.
Spiral CT with VRT. To limit radiation exposure, each volunteer was scanned only once in one of the following designated positions: Supine (49-year-old female; BMI, 23.9), left lateral (46-year-old male; BMI, 24.6), prone (43-year-old female; BMI, 23.2) and right lateral (40-year-old male; BMI, 22.9). After overnight fasting, each subject drank $500 \mathrm{ml}$ contrast agent $(50 \mathrm{ml}$ iodide dissolved in water, diluted to $300 \mathrm{mg} / \mathrm{ml}$. Taizhou Tianrui Pharmaceutical Co., Ltd.) $5 \mathrm{~min}$ before the examination. Images were obtained with a Somatom Sensation 64 CT scanner (Siemens AG). A CT scan was performed at $7-\mathrm{mm}$ intervals from the mid-esophagus to the symphysis pubis. Reformats were viewed on a dedicated diagnostic IDS7 PACS workstation (Sectra Medical Systems $\mathrm{GmbH}$ ) with multiplanar reformatting. A VRT was applied to produce three-dimensional (3D) stomach images.

UGIE. Only 1 healthy male (59 years old; BMI, 21) undergoing a routine UGIE for early gastric cancer screening was included in the present study. The UGIE examination was performed in the standard left lateral decubitus position.

Data processing. To determine the optimal body position for magnet-controlled capsule endoscopy, gastric angles were used as judgment indexes. As a gastric wall scan-derived 3D model was unavailable, gastric angles were estimated qualitatively based on the available UGI-XR and 3D VRT-derived images. Two-dimensional plain air-barium contrast UGI-XR films showed the complete shape of the stomach and the locations of intragastric barium and air, which were dependent upon gravity. 3D VRT showed a stereo model of the intragastric fluid and air-fluid interface. The cross-angle between the longitudinal axis of the fundus and the longitudinal axis of the gastric body (FB angle), and the cross-angle between the longitudinal axis of the gastric body and the longitudinal axis of the antrum (BA angle), were examined. Each angle was categorized as acute $\left(\leq 90^{\circ}\right)$ or obtuse $\left(>90^{\circ}\right)$ based on a combination of UGI-XR and 3D VRT analysis across dynamic position changes. As a greater angle reduces the level of resistance to capsule movement, the body position with the greatest gastric angle was considered optimal for magnetic capsule endoscopy. To better understand the hindrance of FB and BA angles to intragastric capsule movement, special attention was paid to recording variation in these angles in the subject who underwent UGIE.

To assess external magnet placement during capsule endoscopy, the vertical distances from the surface of the body to distal points of the gastric fundus and antrum were measured in UGI-XR and spiral CT images. These distances represent the maximal distance that the capsule would likely be from the surface of the body. As magnetic strength is inversely related to distance, the surface of the torso with the shortest vertical distance to the distal points of the fundus and antrum was chosen for external magnet placement.

Statistical analysis. Descriptions of observations were applied to non-numerical indexes. Each individual subject's numerical data are reported directly; group data are presented as the mean and standard deviation. Vertical distances from the body surface to the distal gastric fundus/antrum of four directions in the same position were compared with 
one-way ANOVA followed by the Student-Newman-Keuls post hoc test. Two-tailed P-values of $<0.05$ were considered significant. All analyses were performed in SPSS version 4.0 (SPSS, Inc.).

\section{Results}

Position of the stomach in different positions. All 10 volunteers completed their examinations without any adverse reactions. Plain film images and 3D models of the stomach in the various decubitus positions are shown in Fig. 1. When the subject was in a supine position, the stomach was horizontal and located in the left hypochondrium (fundus, body), with the antrum extending into the epigastric region. When the subject was lying on either side, the stomach had a tilted orientation, with the fundus being the lowest point near the spine and the antrum being the highest point near the ventral wall.

Plain film images of the stomach from the ventral, dorsal, right side, left side and upright positions are shown in Fig. 2. Compared with the supine position, the stomach in standing subjects had shifted from a largely horizontal position to a vertical position, descending to near the ilium, and in one case even into the pelvis. When subjects moved from lying supine to standing, the shape and location of the stomach shifted, with the light gas ascending gradually and the heavy contrast agent descending. The magnitude of gas/contrast separation differed across the position as follows (from least to most difference): Supine, left lateral, prone, right lateral and standing (The arrows showed the magnitude of gas/contrast separation in Figs. 1 and 2).

Gastric angles and optimal body position. The FB angle was sharply acute in the supine position, slightly wider, though still acute, in the left lateral position, and slightly obtuse in the prone position and the right lateral position. The FB angle flattened instantly to almost $180^{\circ}$ when the subject reached a standing position, wherein the longitudinal axis of the fundus was nearly overlapping with the longitudinal body axis. The BA angle was acute in all four decubitus positions and obtuse in only the standing position. Given the expectation that tighter FB and BA angles would be associated with more resistance to capsule movement, the supine position and left lateral position, which are used in traditional gastroscopy, were ruled out as potentially suitable positions. Among the three remaining candidate positions the prone position was least suitable, due to its relatively small FB angle and its inconvenience and discomfort for the person being examined. The optimal position for magnetic capsule endoscopy was therefore determined to be standing, and the second most optimal was determined to be the right lateral decubitus position.

Vertical distances and external magnet placement. The vertical distances from the body surface to distal points of the fundus and antrum are shown in Tables I and II. The left lower lateral chest surface and ventral wall had the shortest possible distance to the fundus and antrum of any superficial body site in the same position. This difference was statistically significant. Given the expectation that a shorter distance would be associated with greater magnetic strength, suitable
A

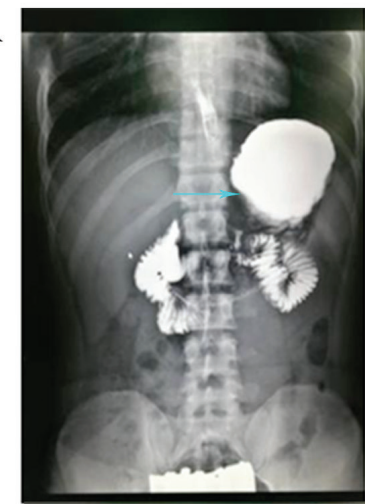

C

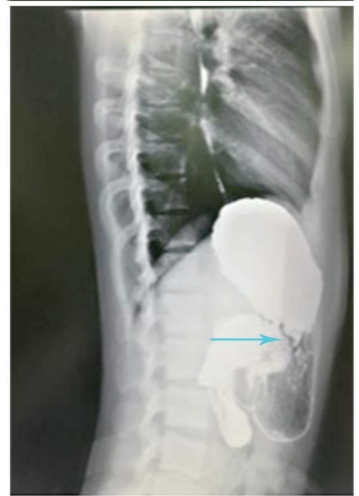

E

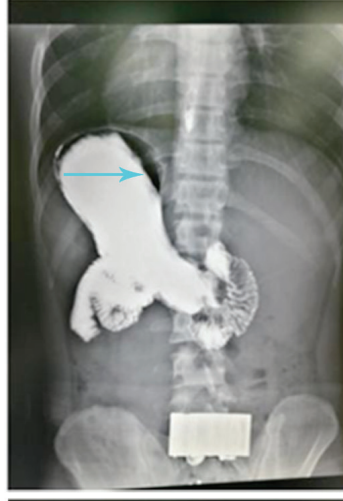

G

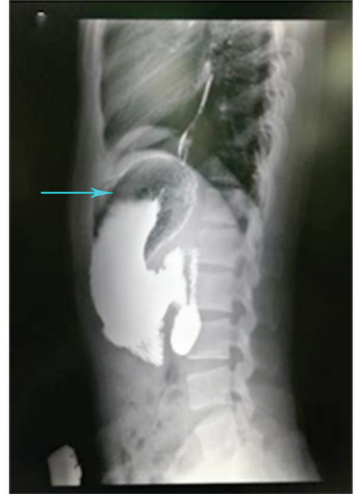

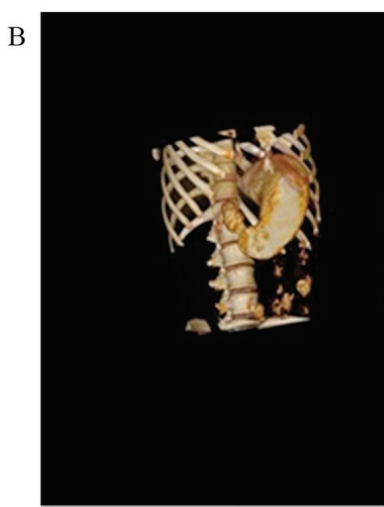

$\mathrm{D}$

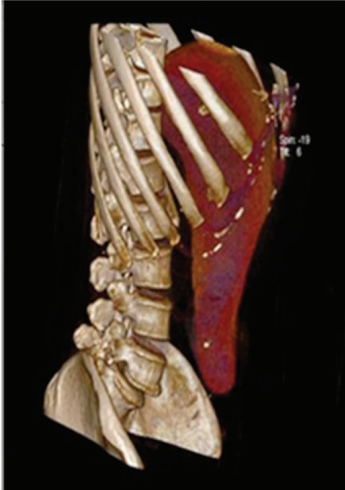

F

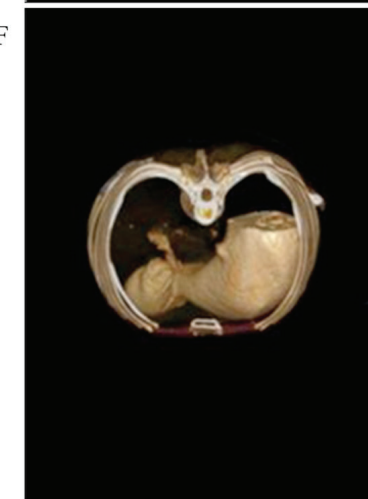

$\mathrm{H}$

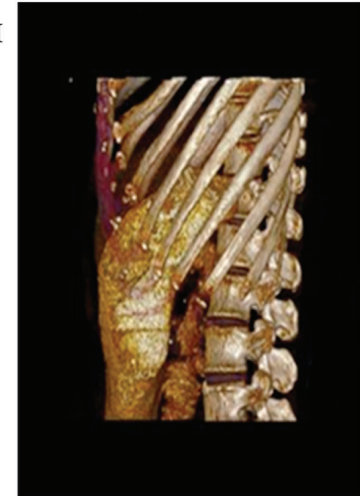

Figure 1. Representative UGI-XR and 3D volume reconstruction images of the stomach with the subject in a decubitus state. (A) UGI-XR and (B) 3D volume reconstruction with the subject in a supine position. (C) UGI-XR and (D) 3D volume reconstruction with the subject on their left side. (E) UGI-XR and (F) 3D volume reconstruction with the subject in a prone position. (G) UGI-XR and (H) 3D volume reconstruction with the subject on their right side. Arrows indicate the magnitude of gas/contrast separation. UGI-XR, upper gastrointestinal X-ray radiography; 3D, three-dimensional.

placement positions for the external magnet included the left lower lateral chest and the presently used ventral wall. 

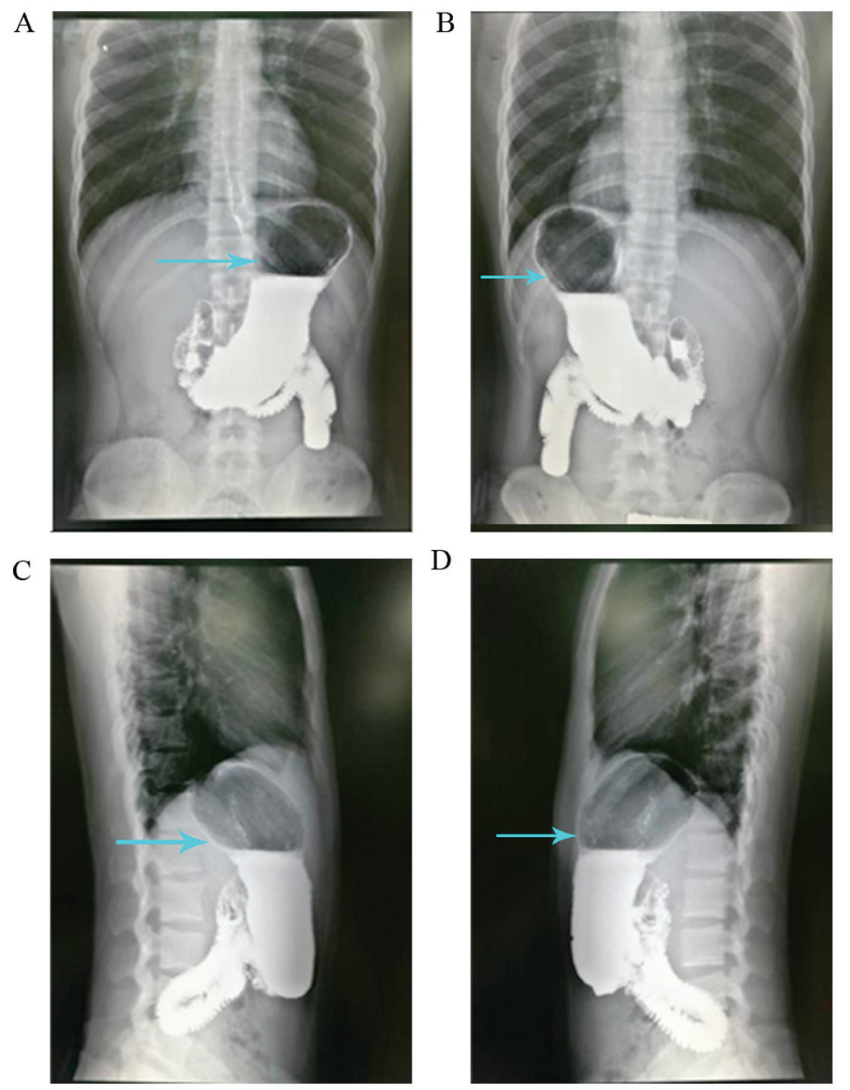

Figure 2. UGI-XR images obtained from an individual standing subject. (A) Posterior-anterior view. (B) Left lateral view. (C) Anterior-posterior view. (D) Right lateral view. Arrows indicate the magnitude of gas/contrast separation. UGI-XR, upper gastrointestinal X-ray radiography.

UGIE. UGIE performed on a 56-year-old healthy volunteer in a routine left lateral position, with special attention being paid to the FB angle and BA angle, revealed a ridged fundus-body junction, with an acute FB angle when the endoscope passed through the cardia (Fig. 3). As air continued to be injected, inflating the stomach cavity, the FB angle widened gradually from sharply acute to obtuse. When the endoscope reached the distal body, the steep slope-like body-antrum junction had an acute BA angle; the junction flattened as additional air was injected, underscoring the importance of inflating the gastric cavity fully to facilitate capsule movement under the control of an external magnet.

\section{Discussion}

The analysis of the data obtained by UGI-XR, spiral CT with VRT and UGIE in the present study showed that, of the positions examined, the best position for a patient to be in during magnet-controlled capsule endoscopy was standing, followed by the right lateral decubitus position. The commonly used supine position was found to be relatively disadvantageous. Additionally, the analysis indicated that it would be beneficial to place the external controlling magnet on the left lateral lower chest in addition to the common placement position of the abdominal wall.

There are three sets of movements involved in UGIE, each with particular purposes, as follows: i) Maintenance of moderate gastric inflation by air injection and deflation;
A
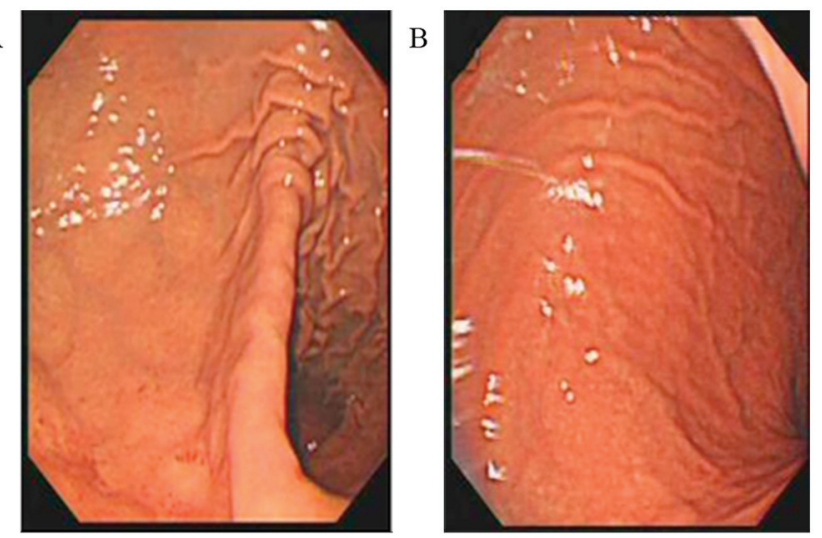

$\mathrm{C}$
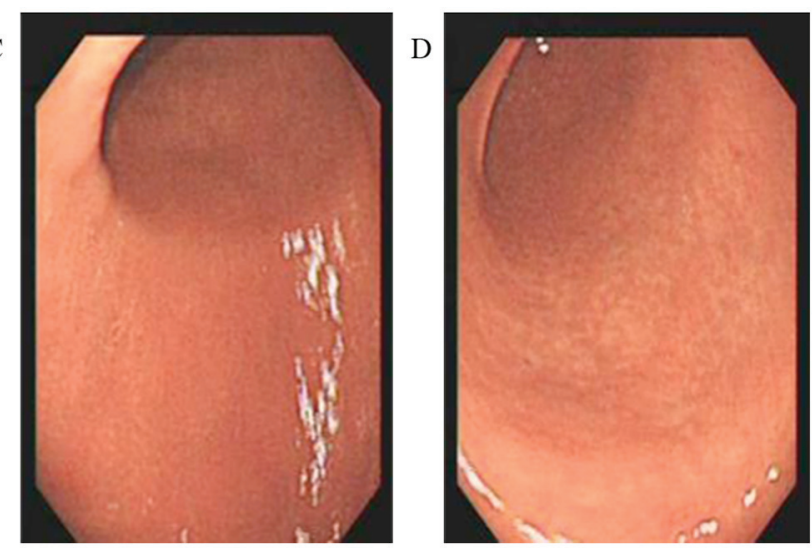

Figure 3. Gastric angles viewed endoscopically. FB angles (A) before inflation and (B) after inflation by air injection. BA angles (C) before inflation and (D) after inflation by air injection. BA, body-to-antrum; FB, fundus-to-body.

ii) keeping the visual field clean with suctioning and washing; and iii) reaching a target site through scope advancement, retreat, and rotation (14). Correspondingly, magnet-controlled capsule endoscopy requires minimal obstacles to capsule movement, clear visual imaging and sufficient magnetic force to move the capsule (5).

Magnetic force is determined directly by magnetic flux density and affected inversely by distance (8). The intensity of the external magnet should be maximized to ensure that there is sufficient magnetic power for capsule control. Magnetic power at a given magnetic induction intensity is maximized by minimizing the distance between the capsule target region and the controlling magnet. The gastric antrum and body are near the abdominal wall, whereas the gastric fundus is near the left lateral lower chest. Therefore, the anterior abdominal wall is a good location for external magnet placement, especially when the goal of the examination is to explore the gastric antrum and body. However, this placement is not well suited for exploring the fundus and cardia; the left lateral lower chest is a better magnet placement site for optimal control of the capsule in the fundus and cardia. Indeed, several reports have attributed fundus/cardia exploration failures to a weak magnetic force $(6,7)$.

Currently, impedance of intragastric capsule mobility by gastric angles is dealt with by inflating the stomach with water and gas-generating reagents $(12,13)$. However, patient 
Table I. Vertical distances from the surface of the torso to the distal gastric fundus.

A, Mean vertical distance to torso surface determined by UGI-XR, cm

\begin{tabular}{lccccc}
\hline & \multicolumn{4}{c}{ Body position } \\
\cline { 2 - 6 } Torso surface & Supine & Left side & Prone & Right side & Standing upright \\
\hline Ventral & $\mathrm{ND}^{\mathrm{b}}$ & $16.3 \pm 3.0$ & $\mathrm{ND}$ & $14.0 \pm 3.0$ & $15.5 \pm 2.6$ \\
Dorsal & $\mathrm{ND}$ & $15.3 \pm 4.6$ & $\mathrm{ND}$ & $19.3 \pm 1.8$ & $16.9 \pm 1.4$ \\
Left lateral & $13.0 \pm 1.4^{\mathrm{a}}$ & $\mathrm{ND}$ & $12.5 \pm 1.4^{\mathrm{a}}$ & $\mathrm{ND}$ & $12.0 \pm 1.5^{\mathrm{a}}$ \\
Right lateral & $28.2 \pm 2.8$ & $\mathrm{ND}$ & $26.6 \pm 4.0$ & $\mathrm{ND}$ & $26.3 \pm 2.3$ \\
\hline
\end{tabular}

$\mathrm{B}$, Vertical distance to torso surface determined by $\mathrm{CT}, \mathrm{cm}$

\begin{tabular}{|c|c|c|c|c|c|}
\hline \multirow[b]{2}{*}{ Torso surface } & \multicolumn{5}{|c|}{ Body position } \\
\hline & Supine & Left side & Prone & Right side & Standing upright \\
\hline Ventral & 15.16 & 15.67 & ND & 13.42 & ND \\
\hline Dorsal & 13.05 & 14.93 & ND & 16.11 & ND \\
\hline Left lateral & 10.17 & 12.51 & ND & 9.13 & ND \\
\hline Right lateral & 24.60 & 23.81 & ND & 23.52 & ND \\
\hline
\end{tabular}

${ }^{\mathrm{a}} \mathrm{P}<0.05$ vs. right lateral position UGI-XR. ${ }^{\mathrm{b}}$ Limitations associated with X-ray meant that only two sets of data were collected for each position except for standing. CT, computed tomography; ND, not determined; UGI-XR, upper gastrointestinal X-ray radiography.

Table II. Vertical distances from the surface of the torso to the distal gastric antrum.

A, Mean vertical distance to torso surface determined by UGI-XR, cm

\begin{tabular}{lccccc}
\hline & \multicolumn{4}{c}{ Body position } \\
\cline { 2 - 6 } Torso surface & Supine & Left side & Prone & Right side & Standing upright \\
\hline Ventral & $\mathrm{ND}^{\mathrm{b}}$ & $8.1 \pm 1.4^{\mathrm{a}}$ & $\mathrm{ND}$ & $8.6 \pm 0.4^{\mathrm{a}}$ & $6.8 \pm 1.2^{\mathrm{a}}$ \\
Dorsal & $\mathrm{ND}$ & $18.1 \pm 3.6$ & $\mathrm{ND}$ & $16.3 \pm 3.2$ & $19.5 \pm 2.4$ \\
Left lateral & $17.2 \pm 3.8$ & $\mathrm{ND}$ & $15.6 \pm 2.3$ & $\mathrm{ND}$ & $14.5 \pm 4.3$ \\
Right lateral & $14.8 \pm 1.2$ & $\mathrm{ND}$ & $14.6 \pm 0.6$ & $\mathrm{ND}$ & $13.0 \pm 1.5$ \\
\hline
\end{tabular}

B, Vertical distance to torso surface determined by CT, $\mathrm{cm}$

\begin{tabular}{|c|c|c|c|c|c|}
\hline \multirow[b]{2}{*}{ Torso surface } & \multicolumn{5}{|c|}{ Body position } \\
\hline & Supine & Left side & Prone & Right side & Standing upright \\
\hline Ventral & 7.06 & 5.53 & ND & 7.65 & ND \\
\hline Dorsal & 15.57 & 17.86 & ND & 17.17 & ND \\
\hline Left lateral & 19.13 & 12.89 & ND & 16.19 & ND \\
\hline Right lateral & 9.94 & 9.47 & ND & 8.33 & ND \\
\hline
\end{tabular}

${ }^{\mathrm{a} P}<0.05$ vs. dorsal position UGI-XR. ${ }^{b}$ Limitations associated with X-ray meant that only two sets of data were collected for each position except for standing. CT, computed tomography; ND, not determined; UGI-XR, upper gastrointestinal X-ray radiography.

tolerance of inflation is limited (13). Belching and gastric emptying also lead to continual loss of inflation $(13,15)$. In the present study, gastric angles were found to differ in relation to body position owing to gravity effects. Notably, the supine position, which is in common use, emerged as the worst position of the five studied positions due to its association with 
sharp acute BF and BA angles. Conversely, in the standing position, the FB and BA angles are extended, placing the fundus, body and antrum in a nearly linear relationship, which is highly amenable to capsule movement. Among the four decubitus positions examined, the right lateral position was found to be the preferred choice owing to its associated relatively wide FA angle.

Regarding visualization clarity, the currently used standard methods of patient fasting with ingestion of deforming agents are far from satisfactory. The challenge of optimizing visualization clarity, however, was beyond the scope of the present modeling study.

The findings of the present analysis and UGIE case observation suggest several possible reasons for the flexibility reported in the first human trial of magnet-controlled capsule endoscopy not being replicated thereafter. In the first trial, capsule endoscopy was performed concomitantly with traditional UGIE with the subject lying on the left side. There was ongoing air injection to maintain gastric inflation, which tends to diminish the FB angle, thereby facilitating capsule movement (5). Conversely, inflation of the stomach in the subsequent studies relied on the subject drinking water with gas-producing agents, which is less effective than active air injection. Hence, capsule movement may have been hindered by a non-extended FB angle in the subsequent studies.

There were several limitations to the present study. First, 3D VRT was not conducted in the standing position due to the technical limitations of CT. 3D sonography in the standing position was attempted as an alternative, but it was not possible to complete volume reconstruction, as the intragastric fluid volume was too great for single-point scanning. The lack of these data may lead to a bias in the model analysis. Additionally, gastric angles were estimated qualitatively, rather than measured precisely. Importantly, this limitation, while not ideal, had no effect on the conclusions of the model analysis. The study cohort was also small. In this regard, however, it is important to note that the gastric shape changes associated with the various positions compared in this study are common knowledge among radiologists.

In summary, the UGI-XR, spiral CT, and UGIE results of the present study suggest that magnet-controlled capsule endoscopy should be performed with the subject standing upright if possible, or lying in the right lateral position if the patient is unable to stand for the examination. Additionally, the results of the present study indicate that suitable positions for placement of the external control magnet include the left lower chest in addition to the commonly employed ventral wall placement, particularly for navigation of the gastric fundus and cardia. It should be pointed out that this was a model analysis study and further validation studies with both animals and humans are warranted.

\section{Acknowledgements}

Not applicable.

\section{Funding}

No funding was received.

\section{Availability of data and materials}

The datasets used and/or analyzed during the present study are available from the corresponding author on reasonable request.

\section{Authors' contributions}

HDZ conceived and designed the study. TJS and CSC were responsible for the collection and analysis of patient data. HDZ revised the manuscript critically for important intellectual content. All authors read and approved the final manuscript.

\section{Ethics approval and consent to participate}

The study was approved by the Independent Ethics Committee of Nanshan Hospital, Guangdong Medical University. Patients provided written informed consent.

\section{Patient consent for publication}

Written informed consent for publication was obtained from every volunteer.

\section{Competing interests}

The authors declare that they have no competing interests.

\section{References}

1. Iddan G, Meron G, Glukhovsky A and Swain P: Wireless capsule endoscopy. Nature 405: 417, 2000.

2. Iddan GJ and Swain CP: History and development of capsule endoscopy. Gastrointest Endosc Clin N Am 14: 1-9, 2004.

3. Iddan GJ: A short history of the gastrointetinal capsule. In: Atlas of Video Capsule Endoscopy. Keuchel M, Hagenmüller F and Tajiri D (eds). Springer, Berlin, Heidelberg, pp2-3, 2006.

4. Gong F, Swain P and Mills T: Wireless endoscopy. Gastrointest Endosc 51: 725-729, 2000.

5. Swain P, Toor A, Volke F, Keller J, Gerber J, Rabinovitz E and Rothstein RI: Remote magnetic manipulation of a wireless capsule endoscope in the esophagus and stomach of humans (with videos). Gastrointest Endosc 71: 1290-1293, 2010.

6. Keller J,Fibbe C, VolkeF, Gerber J,Mosse A, Reimann-Zawadzk M, Rabinovitz E, Layer P, Schmitt D, Andresen V, et al: Inspection of the human stomach using remote-controlled capsule endoscopy: A feasibility study in healthy volunteers (with videos). Gastrointest Endosc 73: 22-28, 2011.

7. Rahman I, Pioche M, Shim CS, Lee SP, Sung IK, Saurin JC and Patel P: Magnet-assisted capsule endoscopy in the upper GI tract by using a novel navigation system (with video). Gastrointest Endosc 83: 889-895.e1, 2016.

8. Rahman I, Kay M, Bryant T, Pelitari S, Salter S, Dimitrov B and Patel P: Optimizing the performance of magnetic-assisted capsule endoscopy of the upper GI tract using multiplanar CT modelling. Eur J Gastroenterol Hepatol 27: 460-466, 2015.

9. Rey JF, Ogata H, Hosoe N, Ohtsuka K, Ogata N, Ikeda K, Aihara H, Pangtay I, Hibi T, Kudo S and Tajiri H: Feasibility of stomach exploration with a guided capsule endoscope. Endoscopy 42: 541-545, 2010.

10. Rey JF, Ogata H, Hosoe N, Ohtsuka K, Ogata N, Ikeda K, Aihara H, Pangtay I, Hibi T, Kudo SE and Tajiri H: Blinded nonrandomized comparative study of gastric examination with a magnetically guided capsule endoscope and standard videoendoscope. Gastrointest Endosc 75: 373-381, 2012. 
11. Denzer U, Röch T, Hoytat B, Abdel-Hamid M, Hebuterne X, Vanbiervielt G, Filippi J, Ogata H, Hosoe N, Ohtsuka K, et al: Magnetically guided capsule versus conventional gastroscopy for upper abdominal complaints: A prospective blinded study. J Clin Gastroenterol 49: 101-107, 2015.

12. Liao Z, Duan XD, Xin L, Bo LM, Wang XH, Xiao GH, Hu LH, Zhuang SL and Li ZS: Feasibility and safety of magnetic controlled capsule endoscopy system in examination of human stomach: A pilot study in healthy volunteers. J Interv Gastroenterol 2: 155-160, 2012.

13. Liao Z, Hou X, Lin-Hu EQ, Sheng JQ, Ge ZZ, Jiang B, Hou XH, Liu JY, Li Z, Huang QY, et al: Accuracy of magnetically controlled capsule endoscopy, compared with conventional gastroscopy in detection of gastric diseases. Clin Gastroenterol Hepatol 14: 1266-1273.e1, 2016.
14. Martin T, Schwab K and Singh S: Principles of gastrointestinal endoscopy. Surgery 32: 139-144, 2014.

15. Zou WB, Hou XH, Xin L, Liu J, Bo LM, YU GY, Liao Z and Li ZS: Magnetic-controlled capsule endoscopy vs. gastroscopy for gastric diseases: A two center self-controlled comparative trial. Endoscopy 47: 525-528, 2015.

cc) (7) (9) This work is licensed under a Creative Commons

EY NG ND Attribution-NonCommercial-NoDerivatives 4.0 International (CC BY-NC-ND 4.0) License. 